\title{
A Literature Review on Iris Segmentation Techniques for Iris Recognition Systems
}

\author{
Ms. Sruthi.T.K ${ }^{1,}$ Ms. Jini.K.M ${ }^{2}$ \\ Post Graduate Scholar, Computer Science Department, Nehru college of Engineering, India ${ }^{I}$ \\ Assistant Professor, Computer Science Department, Nehru College of Engineering, India ${ }^{2}$
}

\begin{abstract}
A biometric system offers automatic identification of a human being based on the unique feature or characteristic which is being possessed by the individual. The iris segmentation has its own major applications in the field of surveillance as well as in security purposes. The performance of the iris recognition systems depends heavily on segmentation and normalization techniques. A review of various segmentation approaches used in iris recognition is done in this paper. The survey is represented in tabular form for quick reference.
\end{abstract}

Index Terms - iris recognition, biometrics, iris segmentation

\section{Introduction}

In the recent years, drastic improvements have been accomplished in the areas like iris recognition, automated iris segmentation, edge detection, boundary detection etc. Iris recognition is a biometric recognition technology that utilizes the pattern recognition techniques based on the high quality images of iris.. An iris recognition system mainly uses infrared or else the visible light. The systems which are based on near infrared light (NIR) are very common because NIR will not generate reflections that makes iris recognition complex. But, NIR images lacks pigment coloration information, thus recognition algorithms must entirely depend on the patterns that are unrelated to color. Iris recognition which is based on visible light raise up pigmentation, thus the recognition systems will be able to exploit the color patterns, which make identification much easier. This is because, the pigmentation patterns contain a lots of information that can be utilized for recognition. But the visible light reflections in these types of systems can result in a extensive amount of noise in the gathered images. A typical iris recognition system consists of mainly three modules. They are image acquisition, preprocessing stage as well as feature extraction and encoding. Image acquisition is a module which involves the capturing of iris images with the help of sensors. Pre-processing module provides the determination of the boundary of iris

within the eye image, and then extracts the iris portion from the image in order to facilitates its processing. It involves the stages like iris segmentation, iris normalization, image enhancement etc. The performance of the system has been analyzed in the feature extraction and encoding stage. All these stages involve their own developments. Major improvements have been made in the field of iris segmentation. In this paper, some of the methods involving iris segmentation have been analyzed.

Approaches like Integrodifferential operator [1], Hough transform [2] constitute a major part in the iris recognition techniques. Many other iris recognition as well as the iris segmentation approaches $[3,4,5,6,7,8]$ has been introduced. Each one has its own advantage as well as the disadvantage. Major drawbacks involved in all the papers is that, the segmentation accuracy has not been achieved yet. Thus, there is a strong need to develop a new segmentation approach that is more reliable as well as robust, which can automatically segment non ideal iris images, which has been acquired using visible illumination in very less constrained imaging environments. Thus, here a unified framework approach [9] has been introduced, which automatically provide the localized eye images from face images for iris recognition. And also, an efficient post processing operations has been introduced in order to mitigate the noisy pixels which have been formed by the misclassification.

\section{A. Integrodifferential operator}

\section{Approaches Used For Iris Recognition}

This approach [1] is regarded as one of the most cited approach in the survey of iris recognition. Daugman uses an integrodifferential operator for segmenting the iris. It find both inner and the outer boundaries of the iris region. The outer as well as the inner boundaries are referred to as limbic and pupil boundaries. The parameters such as the center and radius of the circular boundaries are being searched in the three dimensional parametric space in order to maximize the evaluation functions involved in the the model. This algorithm achieves high performance in iris recognition. It is having a drawback that, it suffers from heavy computation. 


\section{B. Hough Transform}

In this paper, in order to perform personal identification and also the verification, an automated iris recognition system has been examined as the biometrically based technology. This paper also defines the technical issues which are being produced while designing an iris recognition system. The technical issues involve three parts viz. while image acquisition, iris localization and also matching of the extracted iris pattern. The major challenges involved in the image acquisition are in terms of image resolution and also in the focus using some standard optics. Here, an extant system has been used in order to provide a remedy to these challenges. Next issue is the iris localization. That is the image capture during the image acquisition will be a very larger image which contains iris as a part of it. Thus localization of the part that corresponds to the iris from acquired image is very much important. The edge detection has been performed through the gradient-based Canny edge detector, which is followed by the circular Hough transform [2], which is used for iris localization. The final issue is the pattern matching. After the localization of the region of the acquired image which corresponds to the iris, the final operation is to decide whether pattern matches with the previously saved iris pattern. This stage involves alignment, representation, goodness of match and also the decision. All these pattern matching approaches relies mainly on the method which are closely coupled to the recorded image intensities. If there occurs a greater variation in any one of the iris, one way to deal with this is the extraction as well as matching the sets of features that are estimated to be more vigorous to both photometric as well as geometric distortions in the obtained images. The advantage of this method is that it provides segmentation accuracy up to an extent. The drawback of this approach is that, it does not provide any attention to eyelid localization (EL), reflections, eyelashes, and shadows.

\section{Masek Method}

Masek introduced an open iris recognition system [3] for the verification of human iris uniqueness and also its performance as the biometrics. The iris recognition system consists of an automated segmentation system, which localise the iris region from an eye image and also isolate the eyelid, eyelash as well as the reflection regions. This Automatic segmentation was achieved through the utilization of the circular Hough transform in order to localise the iris as well as the pupil regions, and the linear Hough transform has been used for localising the eyelid occlusion. Thresholding has been employed for isolating the eyelashes as well as the reflections. Now, the segmented iris region has got normalized in order to eliminate the dimensional inconsistencies between the iris regions. This was achieved by applying a version of Daugman's rubber sheet model, in which the iris is modeled as a flexible rubber sheet, which is unpacked into a rectangular block with constant polar dimensions. Ultimately, the iris features were encoded by convolving the normalized iris region with the 1D Log-Gabor filters and phase quantizing the output to produce a bit-wise biometric template. For metric matching, the Hamming distance has been chosen, which provides a measure of number of disagreed bits between two templates. The drawback of [2] has been recovered in this paper i.e., the localisation of the circular iris as well as the pupil region, occlusion of eyelids as well as the eyelashes, and also the reflection occurs. The drawback of this approach is that the iris segmentation is not that much accurate and also the speed of the system is low.

\section{Fuzzy clustering algorithm}

A new iris segmentation approach, which has a robust performance in the attendance of heterogeneous as well as noisy images, has been developed in this. The process starts with the image-feature extraction where three discrete i.e., (x, y) which corresponds to the pixel position, and $\mathrm{z}$ which corresponds to its intensity values has got extracted for each and every image pixel, which is followed by the application of a clustering algorithm which is the fuzzy K-means algorithm[4]. This has been used inorder to classify each and every pixel and then generate the intermediate image. This correspondent image is then used by the edge-detector algorithm. As it has additional homogeneous characteristics, this eases the tuning of the parameters which were needed by the edge-detector algorithm. The main advantage of this method is that, it provides a better segmentation for non cooperative iris recognition. The major drawback in this method is that thorough (extensive) search is needed in order to recognize the circle parameters of both the pupil as well as the iris boundaries.

\section{E. Pulling and Pushing (PP) Method}

A perfect (accurate) as well as a rapid iris segmentation algorithm for iris biometrics has been developed in this. There are mainly five major contributions in this. Firstly, a novel reflection removal method has been developed in order to exclude the specularities involved in the input images, also an Adaboost-cascade iris detector has been used in order to detect the iris in them and also to exclude the non iris image parts before further processing such that redundant computations can be avoided. In addition to this, a rough iris center has been extracted in the iris images. Second contribution is that, beginning from the rough iris center, a novel puling and pushing (PP) [5] procedure has been developed in order to accurately localize the circular iris 
boundaries. The PP method directly finds the shortest path to the valid parameters. Third is that, a cubic smoothing spline has been adopted in order to deal with the noncircular iris boundaries. Fourth contribution is that, an efficient method for the localization of the eyelids has been developed. The main difficulties of eyelid localization difficulties such as sharp irregularity of eyelids as well as the noise due to the eyelashes has been addressed proficiently by a rank filter and also a histogram filter. Finally, the eyelashes as well as the shadows have been detected with statistically learned prediction model. The advantage of PP method is the accuracy and speed. The drawback of this method is that the occurrence of the segmentation error.

\section{F. Eight-neighbor connection based clustering}

An efficient as well as robust algorithm for noisy iris image segmentation in the background of non cooperative and less-cooperative iris recognition has been developed in this. The major contributions involved in this are as follows. Firstly, a novel region growing scheme known as the eight-neighbor connection based clustering [6] has been proposed in order to cluster the whole iris image into different parts. Then, genuine iris region has been extracted with the aid of several semantic priors, and also the non-iris regions such as eyelashes, eyebrow, glass frame, hair, etc are identified and also excluded as well, which intensely reduces the possibility of mis localizations occurring on the non-iris regions. Secondly, an integrodifferential constellation has been introduced inorder to accelerate the traditional integrodifferential operator, and then, enhance its global convergence ability for pupillary as well as the limbic boundary localization. Thirdly, a 1-D horizontal rank filter as well as an eyelid curvature model has been adopted in order to tackle the eyelashes as well as the shape irregularity, during eyelid localization. Finally, the eyelash as well as the shadow occlusions has been detected with the aid of learned prediction model which is based on the intensity statistics between different iris regions. The advantage of this method is that the iris segmentation accuracy has been attained. The drawback is segmentation of noisy iris images should be improved.

\section{G. $\quad$ Segmentation approach based on Fourier spectral density}

A new segmentation method for noisy frontal view iris images which is captured with minimum cooperation based on the Fourier spectral density [7] has been proposed in this paper. This approach computes the Fourier spectral density for each pixel with the aid of its neighborhood and then executes row-wise adaptive thresholding, and thus results in a binary image which gives the iris region in a fairly accurate manner. This segmentation method can also be used in order to calculate limbic as well as the pupil boundaries. This is having the advantage that, this has lower computations as compared to that of [1], [2]. The drawback is that, limbic boundary detection should be improved.

\section{H. Circular Gabor Filter}

Segmentation of iris is one of the most vital as well as difficult task in iris recognition. [1] and [2] are widely used for iris texture segmentation. But their computational time consumption is high. Time consumption is mainly required in order to recognize the initial centers of both iris as well as pupil circular boundaries. A simple solution for this problem has been given in this paper. The circular Gabor filter (CGF) [8] has been utilized in order to localize the initial pupil center. The main advantage of this approach is that the segmentation accuracy for both the pupil as well as the iris boundaries has been achieved. The drawback is that, the overall segmentation accuracy is less than that of [9].

All these techniques have improved the performance of the iris recognition system. All these techniques acquire segmentation accuracy in many areas such as boundary detection, iris detection, pupil and limbic boundary detection etc. But none of these papers provide a solution for attaining overall segmentation accuracy. Thus there is a an extreme need for developing a new segmentation method, which are more reliable as well as robust for automatically segmenting the non ideal iris images.

This paper introduces a unified solution for iris segmentation [9]. Here, a new iris segmentation framework has been developed, which can robustly segment the iris images attained using NIR or else the visible illumination. This approach exploits multiple higher order local pixel dependencies using Zernike moments (ZM), in order to strongly (robustly) classify the eye region pixels into iris or else the non iris regions using trained neural network/ support vector machine (NN/SVM) classifiers. Image enhancement using single scale retinex (SSR) algorithm has been employed for illumination variation problem. Face as well as the eye detection modules has been integrated in the unified framework to automatically provide the localized eye region from facial image for segmenting the iris. A robust post processing operations algorithm has been developed inorder to effectively alleviate the noisy pixels which has been caused due to misclassification. 
Table1: Comparison table on literature survey

\begin{tabular}{|c|c|c|c|}
\hline NAME & METHOD & PERFORMANCE & DISADVANTAGES \\
\hline $\begin{array}{l}\text { High confidence } \\
\text { visual recognition of } \\
\text { persons by a test of } \\
\text { statistical } \\
\text { independence }\end{array}$ & $\begin{array}{l}\text { Integrodifferential } \\
\text { operator }\end{array}$ & $\begin{array}{l}\text { High performance in } \\
\text { iris recognition }\end{array}$ & $\begin{array}{l}\text { Computational time is } \\
\text { very high }\end{array}$ \\
\hline $\begin{array}{l}\text { Iris Recognition: An } \\
\text { emerging biometric } \\
\text { technology }\end{array}$ & Hough transform & $\begin{array}{l}\text { Segmentation } \\
\text { accuracy achieved } \\
\text { up to an extent }\end{array}$ & $\begin{array}{l}\text { Does not provide } \\
\text { attention to EL as well } \\
\text { as reflections etc }\end{array}$ \\
\hline $\begin{array}{l}\text { Recognition of } \\
\text { Human Iris Patterns } \\
\text { for Biometric } \\
\text { Identification }\end{array}$ & $\begin{array}{l}\text { Liber Masek's } \\
\text { encoding algorithm }\end{array}$ & $\begin{array}{l}\text { Localization of } \\
\text { circular iris region } \\
\text { as well as eyelids, } \\
\text { eyelashes and also } \\
\text { the reflections } \\
\text { occurs }\end{array}$ & $\begin{array}{l}\text { Speed of the system is } \\
\text { low }\end{array}$ \\
\hline $\begin{array}{l}\text { Iris segmentation } \\
\text { methodology for } \\
\text { non cooperative } \\
\text { recognition }\end{array}$ & $\begin{array}{l}\text { Fuzzy clustering } \\
\text { algorithm }\end{array}$ & $\begin{array}{l}\text { Better segmentation } \\
\text { for non co-operative } \\
\text { iris recognition }\end{array}$ & $\begin{array}{l}\text { Thorough search is } \\
\text { needed to recognize } \\
\text { the circle parameters } \\
\text { of both pupil and iris } \\
\text { boundaries }\end{array}$ \\
\hline $\begin{array}{lr}\text { Toward Accurate } \\
\text { and Fast } & \text { Iris } \\
\text { Segmentation } & \text { for } \\
\text { Iris Biometrics } & \\
\end{array}$ & $\begin{array}{l}\text { Pushing and pulling } \\
\text { (PP) method }\end{array}$ & $\begin{array}{l}\text { Possess accuracy } \\
\text { and speed }\end{array}$ & $\begin{array}{l}\text { Occurrence of } \\
\text { segmentation error }\end{array}$ \\
\hline $\begin{array}{l}\text { Efficient and robust } \\
\text { segmentation of } \\
\text { noisy iris images for } \\
\text { non cooperative } \\
\text { iris recognition }\end{array}$ & $\begin{array}{l}\text { Eight-neighbor } \\
\text { connection based } \\
\text { clustering }\end{array}$ & $\begin{array}{l}\text { iris segmentation } \\
\text { accuracy has been } \\
\text { attained to an extent }\end{array}$ & $\begin{array}{l}\text { segmentation of noisy } \\
\text { iris images should be } \\
\text { improved }\end{array}$ \\
\hline $\begin{array}{l}\text { Efficient } \\
\text { segmentation } \\
\text { technique for noisy } \\
\text { frontal view iris } \\
\text { images using } \\
\text { Fourier spectral } \\
\text { density }\end{array}$ & $\begin{array}{l}\text { Segmentation } \\
\text { approach based on } \\
\text { Fourier spectral } \\
\text { density }\end{array}$ & $\begin{array}{l}\text { Computational } \\
\text { complexity is low }\end{array}$ & $\begin{array}{l}\text { Limbic boundary } \\
\text { detection should be } \\
\text { improved. }\end{array}$ \\
\hline $\begin{array}{l}\text { Iris Segmentation in } \\
\text { Visible Wavelength } \\
\text { Environment }\end{array}$ & $\begin{array}{l}\text { Circular Gabor } \\
\text { Filter }\end{array}$ & $\begin{array}{l}\text { segmentation } \\
\text { accuracy for both } \\
\text { the pupil as well as } \\
\text { the iris boundaries } \\
\text { has been achieved }\end{array}$ & $\begin{array}{l}\text { Over all segmentation } \\
\text { accuracy is less than } \\
\text { that of the unified } \\
\text { framework approach }\end{array}$ \\
\hline
\end{tabular}

\section{Conclusion}

This paper presents a literature survey on the various segmentation techniques involved in iris recognition. There are various techniques that can be used for this purpose. Overall segmentation accuracy of all these techniques has been analyzed. Higher the segmentation rate, thus higher is its performance The unified framework has the highest segmentation rate and also has highest performance. 


\section{References}

[1] J.G. Daugman, High confidence visual recognition of persons by a test of statistical independence, IEEE Transactions on Pattern Analysis and Machine Intelligence, 15, p.1148, Nov 1993

[2] R.P. Wildes, Iris recognition: An emerging biometric technology, Proceedings of the IEEE, 85 p.1348, sep. 1997

[3] L. Masek, "Recognition of Human Iris Patterns for Biometric Identification", M.S. Dissertation, the University of Western Australia, 2003

[4] H. Proença, L.A. Alexandre, "Iris segmentation methodology for non-cooperative recognition", IEE Proceedings of Vision, Image and Signal Processing, pp. 199-205, 2006

[5] Z. He, T. Tan, Z. Sun, and X. Qiu, “Toward accurate and fast iris segmentation for iris biometrics," IEEE Trans. Pattern Anal. Machine Intelligence, vol. 31, no. 9, pp. 1670-1684, Sep. 2009.

[6] T. Tan, Z. He, and Z. Sun, "Efficient and robust segmentation of noisy iris images for non-cooperative iris recognition," Image Vis. Comput., vol. 28, no. 2, pp. 223-230, Feb. 2010.

[7] N.B. Puhan, N. Sudha, A. Sivaraman Kaushalram, Efficient segmentation technique for noisy frontal view iris images using Fourier spectral density, Signal, Image and Video Processing, 5, p. 105, 2011.

[8] Radman, A., Jumari, K., and Zainal, N, Iris segmentation in visible wavelength environment. Procedia Engineering, 41:743-748, 2012

[9] Tan, C. and Kumar, A., Unified framework for automated iris segmentation using distantly acquired face images. IEEE Transactions on Image Processing, 21(9):4068-4079, S 